\title{
Arc Length Inequality for a Certain Class of Analytic Functions Related to Conic Regions
}

\author{
Wasim Ul-Haq, Muhammad Arif, and Asfandyar Khan \\ Department of Mathematics, Abdul Wali Khan University, Mardan, Pakistan \\ Correspondence should be addressed to Wasim Ul-Haq; wasim474@hotmail.com
}

Received 22 May 2013; Accepted 26 July 2013

Academic Editor: Narendra Govil

Copyright (c) 2013 Wasim Ul-Haq et al. This is an open access article distributed under the Creative Commons Attribution License, which permits unrestricted use, distribution, and reproduction in any medium, provided the original work is properly cited.

In our present investigation, we introduce a subclass of analytic function associated with conic regions which is a form of generalized close-to-convexity. The arc-length inequality for a class of analytic function is well known. We derive this inequality for the newly defined class and also study some of its interesting consequences.

\section{Introduction}

Let $\mathscr{A}$ denote the class of functions $f$ :

$$
f(z)=z+\sum_{n=2}^{\infty} a_{n} z^{n},
$$

which are analytic in the unit disc $\mathcal{U}=\{z:|z|<1\}$. Let $\mathcal{S}$ denote the class of all functions in $\mathscr{A}$ which are univalent. Also let $\mathcal{S}^{*}, \mathscr{C}$, and $\mathscr{K}$ be the well-known subclasses of $\mathscr{A}$ consisting of all functions which are, respectively, of starlike, convex, and close-to-convex.

Kanas and Wisniowska $[1,2]$ studied the classes of $k$ uniformly convex denoted by $k-\mathscr{U} \mathscr{C} \mathscr{V}$ and the corresponding class $k-\mathcal{S} \mathscr{T}$ related by the Alexandar type relation. Later Acu [3] considered the class $k$-uniformly close-to-convex denoted by $k-\mathscr{U} \mathscr{K}$ to be defined as

$$
\begin{gathered}
k-\mathscr{U} \mathscr{K}=\left\{f(z) \in \mathscr{A}: \operatorname{Re}\left(\frac{z f^{\prime}(z)}{g(z)}\right)>k\left|\frac{z f^{\prime}(z)}{g(z)}-1\right|,\right. \\
g(z) \in k-\mathcal{S} \mathscr{T}, z \in E\} ;
\end{gathered}
$$

for more detail see [4-6].

In [7], the conic domain $\Omega_{k, \gamma}$ with complex order is defined as

$$
\Omega_{k, \gamma}=\gamma \Omega_{k}+(1-\gamma), \quad 0<\operatorname{Re} \gamma \leq k+1,
$$

where

$$
\Omega_{k}=\left\{u+i v: u>k \sqrt{(u-1)^{2}+v^{2}}\right\} .
$$

The domain $\Omega_{k, \gamma}$ is elliptic for $k>1$, hyperbolic when $0<$ $k<1$, parabolic for $k=1$, and right half plane when $k=0$. The functions which play the role of extremal functions for the conic regions of complex order are given as

$$
\begin{aligned}
& p_{k, \gamma}(z) \\
& \left\{\begin{array}{ll}
\frac{1+(2 \gamma-1) z}{1-z}, & k=0, \\
1+\frac{2 \gamma}{\pi^{2}}\left(\log \frac{1+\sqrt{z}}{1-\sqrt{z}}\right)^{2}, \quad k=1, \\
1+\frac{2 \gamma}{1-k^{2}} \sinh ^{2}\left[\left(\frac{2}{\pi} \arccos k\right) \arctan h \sqrt{z}\right], \\
1+\frac{\gamma}{k^{2}-1} \sin \left(\frac{\pi}{2 R(t)} \int_{0}^{u(z) / \sqrt{t}} \frac{1}{\sqrt{1-x^{2}} \sqrt{1-(t x)^{2}}} d x\right. \\
+\frac{\gamma}{k^{2}-1}, \quad k>1,
\end{array}\right)
\end{aligned}
$$

where $u(z)=(z-\sqrt{t}) /(1-\sqrt{t z}), t \in(0,1), z \in E$, and $z$ is chosen such that $k=\cosh \left(\pi R^{\prime}(t) / 4 R(t)\right)$, where $R(t)$ is the 
Legendre's complete elliptic integral of the first kind and $R^{\prime}(t)$ is complementary integral of $R(t)$, see $[1,2]$.

Let $P=\{p(z): p(0)=1$ and $\operatorname{Re} p(z)>0, z \in E\}$ be the class of functions with positive real part, and let $k-P(\gamma)$ be the subclass of $P$ containing the functions $p(z)$, such that $p(z) \prec p_{k, \gamma}(z)$. Motivated from Noor's work [8], we extend class $k-P(\gamma)$ to class $k-P_{m}(\gamma), m \geq 2$ which is defined as

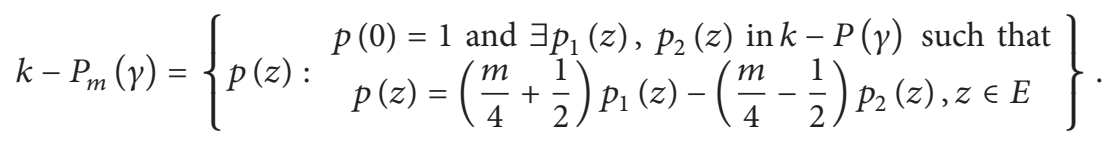

Note that $k-P_{2}(\gamma)=k-P(\gamma)$ and $0-P_{m}(0)=P_{m}$, the class introduced and studied by Pinchuk [9].

We define the following class:

$$
\begin{aligned}
k & -\mathcal{U T}_{m}(\gamma) \\
& =\left\{f(z) \in \mathscr{A}: \frac{f^{\prime}(z)}{g^{\prime}(z)} \in P, g(z) \in k-\mathcal{U V}_{m}(\gamma), z \in E\right\},
\end{aligned}
$$

where

$$
\begin{aligned}
k-\mathscr{U V}_{m}(\gamma) & \\
= & \left\{f(z) \in \mathscr{A}: 1+\frac{z f^{\prime \prime}(z)}{f^{\prime}(z)} \in k-P_{m}(\gamma), z \in E\right\} .
\end{aligned}
$$

Geometrically, a function $f(z) \in \mathscr{U}_{m}(\gamma)$ means that the functional $1+\left(z f^{\prime \prime}(z) / f^{\prime}(z)\right)$ takes all the values in conic domain $\Omega_{k, \gamma}$ and its boundary rotation is at most $m \pi$. We note that class $k-\mathcal{U T}_{m}(\gamma)$ coincides with already known classes of analytic functions by choosing special values for the involved parameters. For example, for $k=0, \gamma=1$, we have the class $\mathscr{T}_{m}$ introduced and studied by Noor [10], and further along with this by taking $m=2$, we obtain the well-known class $\mathscr{K}$ of close-to-convex functions. The purpose of this paper is to investigate some interesting properties of class $k-\mathscr{U} \mathscr{T}_{m}(\gamma)$. For this, we require the following results.

Lemma 1. A function $f \in k-\mathcal{U V}_{m}(\gamma)$ if and only if

(i) $f^{\prime}(z)=\left[f_{1}^{\prime}(z)\right]^{\gamma /(1+k)}, f_{1}(z) \in \mathscr{V}_{m}$,

(ii) there exist two normalized starlike functions $s_{1}(z)$ and $s_{2}(z)$ such that

$$
f^{\prime}(z)=\left[\frac{\left(s_{1}(z) / z\right)^{((k / 4)+(1 / 2))}}{\left(s_{2}(z) / z\right)^{((k / 4)-(1 / 2))}}\right]^{\gamma /(1+k)} .
$$

The above lemma can be proved by using the similar procedure as in [11]; also see [8].

Lemma 2 (see [12]). Let $h \in P$ with $z=r e^{i \theta}$. Then,

$$
\frac{1}{2 \pi} \int_{0}^{2 \pi}|h(z)|^{2} d \theta \leq \frac{1+3 r^{2}}{1-r^{2}}
$$

\section{Some Properties of the Class $k-\mathscr{U} \mathscr{T}_{m}(\gamma)$}

In this section, we provide some of the interesting properties of class $k-\mathscr{U} \mathscr{T}_{m}(\gamma)$ such as radius of convexity problem, arc length, and growth rate of its coefficients. The following theorem is readily seen when we proceed on similar lines as in [13].

Theorem 3. The function $f(z) \in k-\mathscr{U} \mathscr{T}_{m}(\gamma)$ if and only if

$$
f^{\prime}(z)=\frac{\left(f_{1}(z)\right)^{((m / 4)+(1 / 2)) \gamma}}{\left(f_{2}(z)\right)^{((m / 4)-(1 / 2)) \gamma}}
$$

where $f_{1}(z)$ and $f_{2}(z)$ are close-to-convex functions.

Theorem 4. Let $f \in k-\mathcal{U} \mathscr{T}_{m}(\rho, \gamma)$ in E. Then, $f \in C$ for $|z|<r_{0}$, where

$$
r_{0}=\frac{2(1+k)}{m \gamma+2 k+2+\sqrt{[m \gamma+2 k+2]^{2}-4(1+k)(2 \gamma-k-1)}} .
$$

This result is sharp.

Proof. We can write

$$
f^{\prime}(z)=g^{\prime}(z) h(z), \quad g(z) \in k-\mathscr{U}_{m}(\gamma), h(z) \in P .
$$

Using Lemma 1(ii), we get

$$
f^{\prime}(z)=\left[\frac{\left(s_{1}(z) / z\right)^{((m / 4)+(1 / 2))}}{\left(s_{2}(z) / z\right)^{((m / 4)-(1 / 2))}}\right]^{\gamma /(1+k)} h(z),
$$

where $s_{1}$ and $s_{2}$ are starlike functions. Logarithmic differentiation of (14) gives us

$$
\begin{aligned}
& \frac{z f^{\prime \prime}(z)}{f^{\prime}(z)} \\
& \quad=\frac{\gamma}{1+k}\left[-1+\left(\frac{m}{4}+\frac{1}{2}\right) \frac{z s_{1}^{\prime}(z)}{s_{1}(z)}-\left(\frac{m}{4}-\frac{1}{2}\right) \frac{z s_{2}^{\prime}(z)}{s_{2}(z)}\right] \\
& \quad+\frac{z h^{\prime}(z)}{h(z)} .
\end{aligned}
$$


This implies that

$$
\begin{aligned}
& 1+ \frac{z f^{\prime \prime}(z)}{f^{\prime}(z)} \\
&=\frac{1+k-\gamma}{1+k}+\frac{\gamma}{1+k}\left[\left(\frac{m}{4}+\frac{1}{2}\right) \frac{z s_{1}^{\prime}(z)}{s_{1}(z)}\right. \\
& \\
&\left.\quad-\left(\frac{m}{4}-\frac{1}{2}\right) \frac{z s_{2}^{\prime}(z)}{s_{2}(z)}\right]+\frac{z h^{\prime}(z)}{h(z)} .
\end{aligned}
$$

Now using distortion results for the class $P$, we have

$$
\begin{aligned}
& \operatorname{Re}\left(1+\frac{z f^{\prime \prime}(z)}{f^{\prime}(z)}\right) \\
& \geq \frac{1+k-\gamma}{1+k}+\frac{\gamma}{1+k}\left[\left(\frac{m}{4}+\frac{1}{2}\right) \frac{1-r}{1+r}\right. \\
& \left.\quad-\left(\frac{m}{4}-\frac{1}{2}\right) \frac{1+r}{1-r}\right]-\frac{2 r}{1-r^{2}} \\
& =\frac{(1+k-\gamma)\left(1-r^{2}\right)+\gamma\left[1+r^{2}-m r\right]-2 r(1+k)}{(1+k)\left(1-r^{2}\right)} .
\end{aligned}
$$

The right hand side of (17) is positive for $|z|<r_{0}$, where $r_{0}$ is given by (12). The sharpness can be viewed from the function $f_{0} \in k-\mathscr{U T}_{m}(\gamma)$, given by

$$
f_{0}^{\prime}(z)=\frac{(1+z)^{(\gamma /(k+1))(((m / 2)-1)+1)}}{(1-z)^{(\gamma /(k+1))(((m / 2)+1)+1)}}, \quad z \in E .
$$

We note the following interesting special cases:

(i) For $\gamma=1$, we have the radius of convexity for class $k-\mathscr{U}_{m}$.

(ii) For $\gamma=1$ and $k=0$, we have the radius of convexity for class $\mathscr{T}_{m}$, proved by Noor [10].

(iii) For $\gamma=1, k=0$ and $m=2$, we have radius of convexity for close-to-convex functions which is well known.

Theorem 5. Let $f \in k-\mathcal{U} \mathscr{T}_{m}(\gamma)$ with $k \geq 0, m \geq 2$, and $((m+2) /(1+k)) \operatorname{Re} \gamma>1$. Then,

$$
L_{r}(f) \leq A(k, \gamma, m)\left(\frac{1}{1-r}\right)^{(1 / 2)((m+2) /(1+k)) \operatorname{Re} \gamma} .
$$

The exponent $(1 / 2)((m+2) /(1+k)) \operatorname{Re} \gamma$ is sharp.

Proof. Let $f \in k-\mathcal{U}_{m}(\rho, \gamma)$. Then, there exists $g(z) \in k-$ $\mathscr{U} \mathscr{V}_{m}(\gamma)$ such that

$$
f^{\prime}(z)=g^{\prime}(z) h(z), \quad h \in P .
$$

From the definition of $k-\mathscr{U}_{m}(\gamma)$, one can deduce that $g(z) \in k-\mathscr{U}_{\mathscr{V}_{m}}(1)$ implies that $g(z) \in \mathscr{V}_{m}(k /(k+1))$.
Now using (20), Lemma 1(ii), and distortion theorems for starlike functions, we have

$$
\begin{aligned}
L_{r}(f)= & \int_{0}^{2 \pi}\left|z g^{\prime}(z) h(z)\right| d \theta, \\
g(z) \in & k-\mathscr{U} \mathscr{V}_{m}(\gamma), h(z) \in P \\
= & \int_{0}^{2 \pi}\left|z \frac{\left(s_{1}(z) / z\right)^{((m / 4)+(1 / 2))(1 /(1+k)) \gamma}}{\left(s_{2}(z) / z\right)^{((m / 4)-(1 / 2))(1 /(1+k)) \gamma}}\right| h(z) \mid d \theta \\
= & \int_{0}^{2 \pi}\left|z^{1-\gamma(1 /(k+1))} \frac{\left(s_{1}(z)\right)^{((m / 4)+(1 / 2))(1 /(1+k)) \gamma}}{\left(s_{2}(z)\right)^{((m / 4)-(1 / 2))(1 /(1+k)) \gamma}}\right| \\
\leq & \frac{2^{((m / 2)-1)(1 /(k+1)) \operatorname{Re} \gamma}}{r^{((m / 4)+(1 / 2))(1 /(k+1)) \operatorname{Re} \gamma-1}} \\
& \times \int_{0}^{2 \pi}\left|s_{1}(z)\right|^{(1 / 4)((m+2) /(1+k)) \operatorname{Re} \gamma}|h(z)| d \theta .
\end{aligned}
$$

By using Hölder's inequality, this gives

$$
\begin{aligned}
L_{r}(f) \leq & \frac{2^{((m / 2)-1)(1 /(k+1)) \operatorname{Re} \gamma}}{r^{((m / 4)+(1 / 2))(1 /(k+1)) \operatorname{Re} \gamma-1}} \\
& \times\left(\frac{1}{2 \pi} \int_{0}^{2 \pi}\left|s_{1}(z)\right|^{(1 / 2)((m+2) /(1+k)) \operatorname{Re} \gamma} d \theta\right)^{1 / 2} \\
& \times\left(\frac{1}{2 \pi} \int_{0}^{2 \pi}|h(z)|^{2} d \theta\right)^{1 / 2} .
\end{aligned}
$$

Since $((m+2) /(1+k)) \operatorname{Re} \gamma>1$, therefore subordination for starlike functions and Lemma 2 give us

$$
L_{r}(f) \leq A(k, \gamma, m)\left(\frac{1}{1-r}\right)^{(1 / 2)((m+2) /(1+k)) \operatorname{Re} \gamma} .
$$

The function $F_{0}(z) \in k-\mathscr{U} \mathscr{T}_{m}(\gamma)$ is defined by

$$
F_{0}^{\prime}(z)=G_{0}^{\prime}(z) h_{0}(z)
$$

where

$$
G_{0}^{\prime}(z)=\frac{(1+z)^{((m / 2)-1)(1 /(1+k)) \gamma}}{(1-z)^{((m / 2)+1)(1 /(1+k)) \gamma}}, \quad h_{0}(z)=\frac{1+z}{1-z}
$$

shows that the exponent is sharp.

Some special choices in the above theorem give us the following interesting results.

Corollary 6. Let $f \in k-\mathcal{U} \mathscr{T}_{2}(1)$. Then

$$
L_{r}(f) \leq A(k)\left(\frac{1}{1-r}\right)^{2 /(1+k)} .
$$

Corollary 7. Let $f(z) \in \mathscr{T}_{m}$. Then

$$
L_{r}(f) \leq A(m)\left(\frac{1}{1-r}\right)^{(m / 2)+1} .
$$


Coefficient Growth Problems. The problem of growth rate and asymptotic behavior of coefficients is well known. In the next results, we study these problems for class $k-\mathcal{U T}_{m}(\gamma)$ by varying different parameters.

Theorem 8. Let $f \in k-\mathcal{U} \mathscr{T}_{m}(\gamma)$ with $k \geq 0, m \geq 2$ and $((m+2) /(1+k)) \operatorname{Re} \gamma>1$. Then

$$
\left|a_{n}\right|=O(1) n^{((m / 2)+1)(\operatorname{Re} \gamma /(1+k))-1}, \quad(n \longrightarrow \infty) .
$$

The exponent is sharp.

Proof. With $z=r e^{i \theta}$, Cauchy's theorem gives us

$$
n a_{n}=\frac{1}{2 \pi r^{n}} \int_{0}^{2 \pi}\left|z f^{\prime}(z)\right| d \theta=\frac{1}{2 \pi r^{n}} L_{r}(f), \quad z=r e^{i \theta} .
$$

Using Theorem 5 and putting $r=1-(1 / n)$, we obtain the required result. The sharpness follows from the function $F_{0}$ defined by the relation (24).

Corollary 9. Let $f \in k-\mathcal{U} \mathscr{T}_{m}(1)$, and let it be of the form (1). Then, for $n>3, k \geq 2$, one has

$$
\left|a_{n}\right|=O(1) n^{((m / 2)+1)(1 /(1+k))-1} .
$$

For $k=0$, in the above corollary, we have growth rate of coefficients problem for functions in class $\mathscr{T}_{m}$, and, for $k=0$, $m=2$ gives us the growth rate of coefficients for close-to-convex functions, which are well known.

\section{Acknowledgments}

The authors want to acknowledge worthy referees of this paper for their insightful comments which greatly improves the entire presentation of the paper. They would also like to thank Prof. Dr. Ehsan Ali, VC AWKUM, for providing research facilities.

\section{References}

[1] S. Kanas and A. Wisniowska, "Conic regions and $k$-uniform convexity," Journal of Computational and Applied Mathematics, vol. 105, no. 1-2, pp. 327-336, 1999.

[2] S. Kanas and A. Wisniowska, "Conic domains and starlike functions," Revue Roumaine de Mathématiques Pures et Appliquées, vol. 45, no. 4, pp. 647-657, 2000.

[3] M. Acu, "On a subclass of $n$-uniformly close to convex functions," General Mathematics, vol. 14, no. 1, pp. 55-64, 2006.

[4] A. Gangadharan, T. N. Shanmugam, and H. M. Srivastava, "Generalized hypergeometric functions associated with $k$ uniformly convex functions," Computers \& Mathematics with Applications, vol. 44, no. 12, pp. 1515-1526, 2002.

[5] E. Aqlan, J. M. Jahangiri, and S. R. Kulkarni, "New classes of $k$ uniformly convex and starlike functions," Tamkang Journal of Mathematics, vol. 35, no. 3, pp. 261-266, 2004.

[6] S. Kanas and H. M. Srivastava, "Linear operators associated with $k$-uniformly convex functions," Integral Transforms and Special Functions, vol. 9, no. 2, pp. 121-132, 2000.
[7] K. I. Noor, M. Arif, and W. Ul-Haq, "On $k$-uniformly close-toconvex functions of complex order," Applied Mathematics and Computation, vol. 215, no. 2, pp. 629-635, 2009.

[8] K. I. Noor, "On a generalization of uniformly convex and related functions," Computers \& Mathematics with Applications, vol. 61, no. 1, pp. 117-125, 2011.

[9] B. Pinchuk, "Functions of bounded boundary rotation," Israel Journal of Mathematics, vol. 10, pp. 6-16, 1971.

[10] K. I. Noor, "On a generalization of close-to-convexity," International Journal of Mathematics and Mathematical Sciences, vol. 6, no. 2, pp. 327-333, 1983.

[11] M. K. Aouf, "On certain classes of $p$-valent functions," International Journal of Mathematics and Mathematical Sciences, vol. 9, no. 1, pp. 55-64, 1986.

[12] Ch. Pommerenke, "On close-to-convex analytic functions," Transactions of the American Mathematical Society, vol. 114, pp. 176-186, 1965.

[13] K. I. Noor, "On certain analytic functions related with strongly close-to-convex functions," Applied Mathematics and Computation, vol. 197, no. 1, pp. 149-157, 2008. 


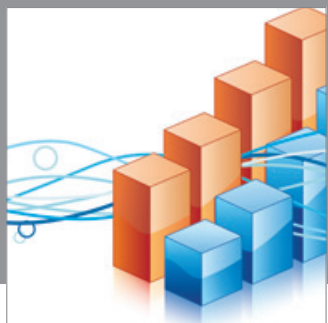

Advances in

Operations Research

mansans

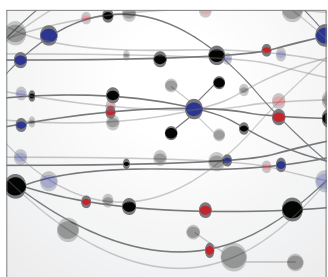

The Scientific World Journal
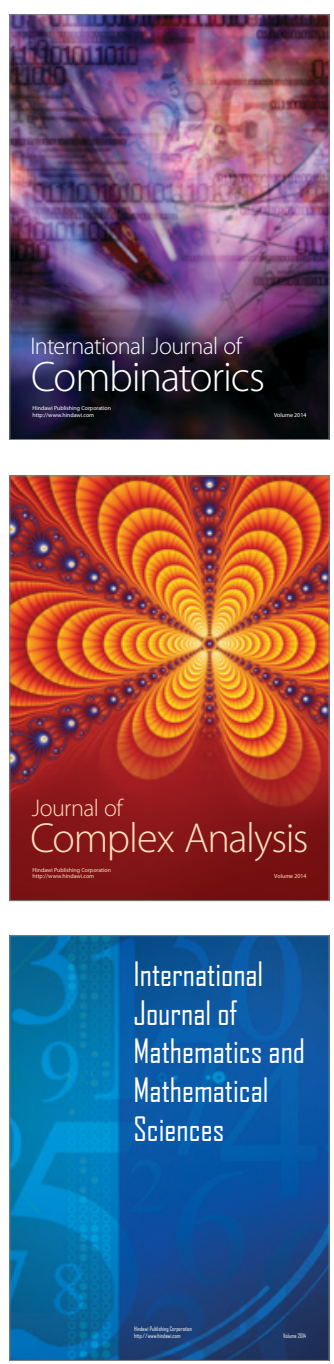
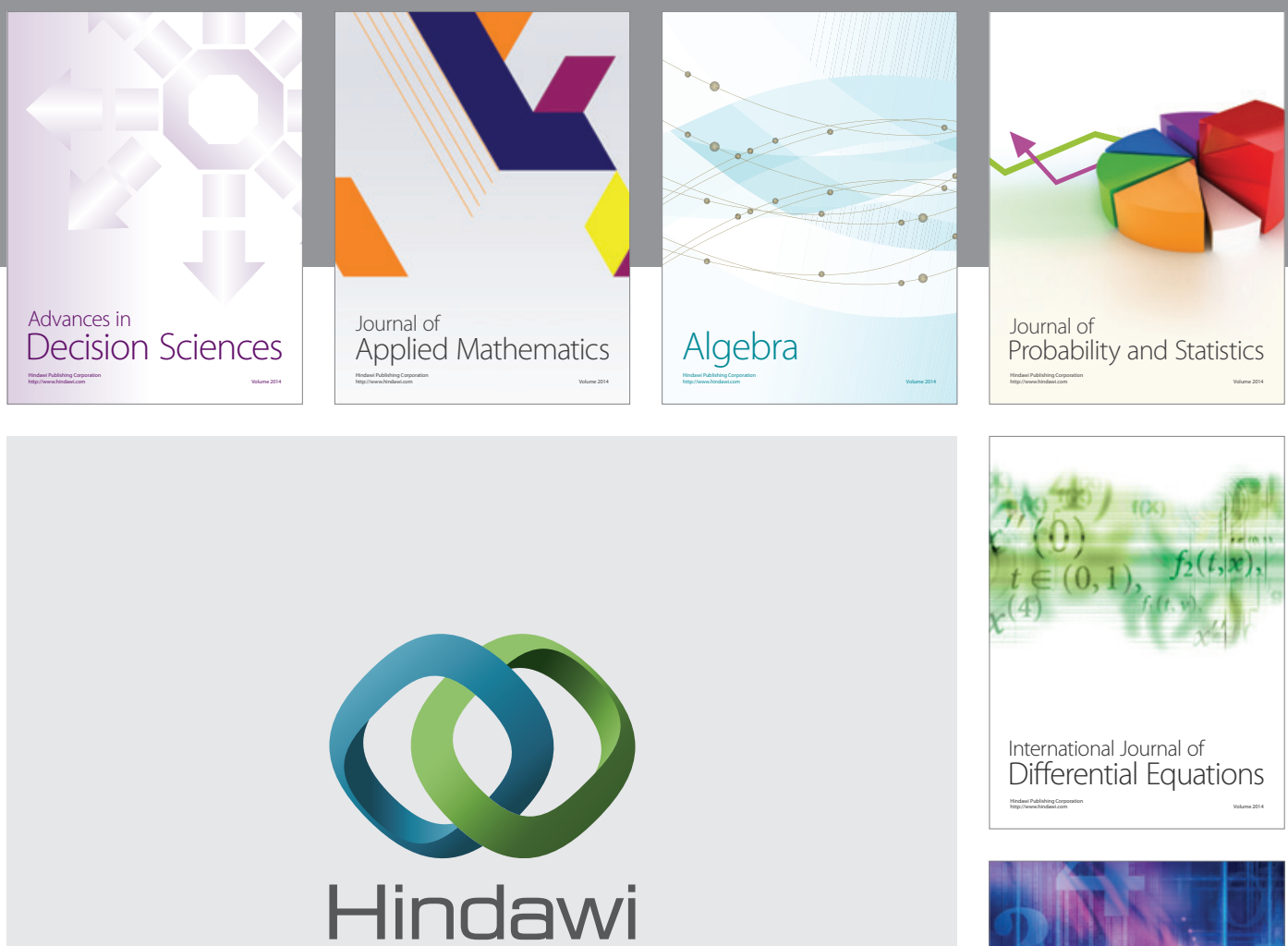

Submit your manuscripts at http://www.hindawi.com
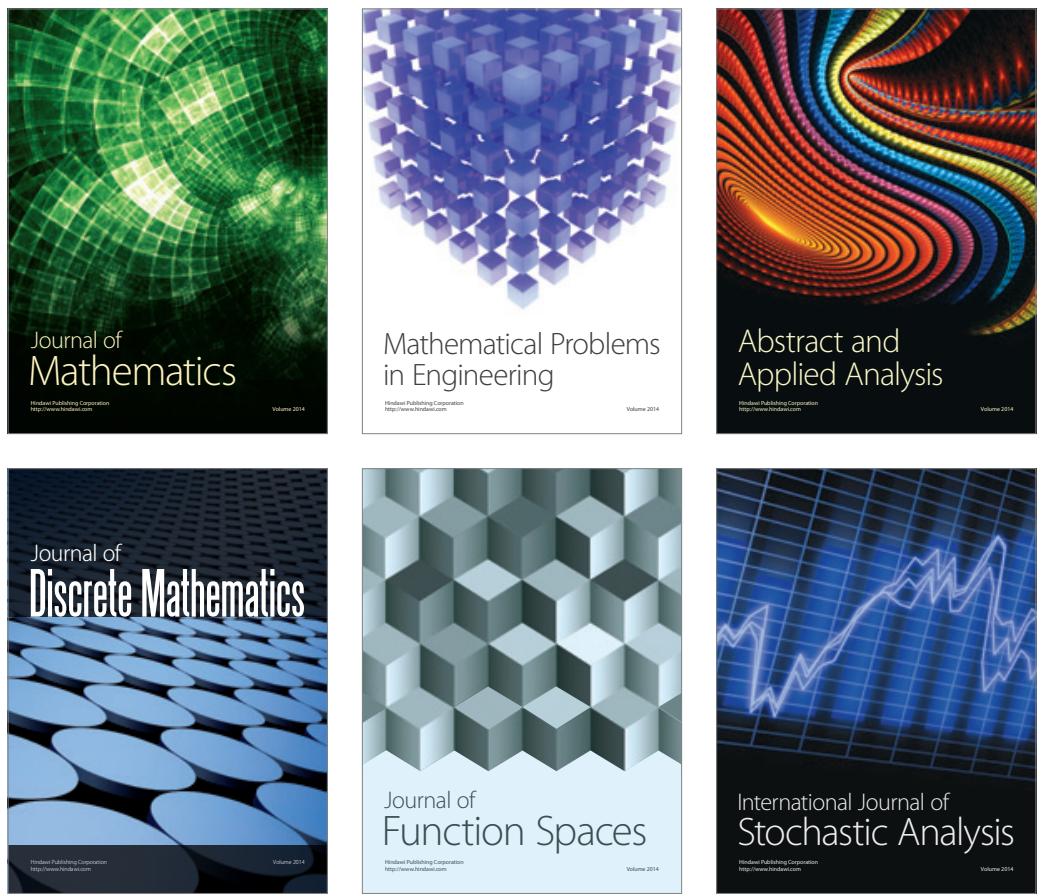

Journal of

Function Spaces

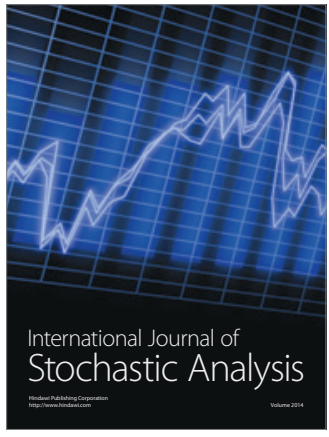

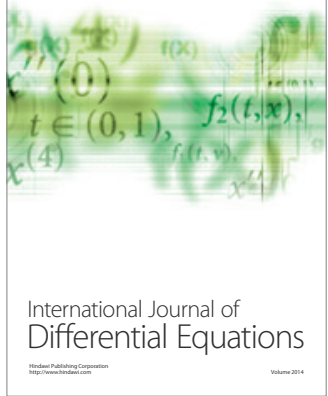
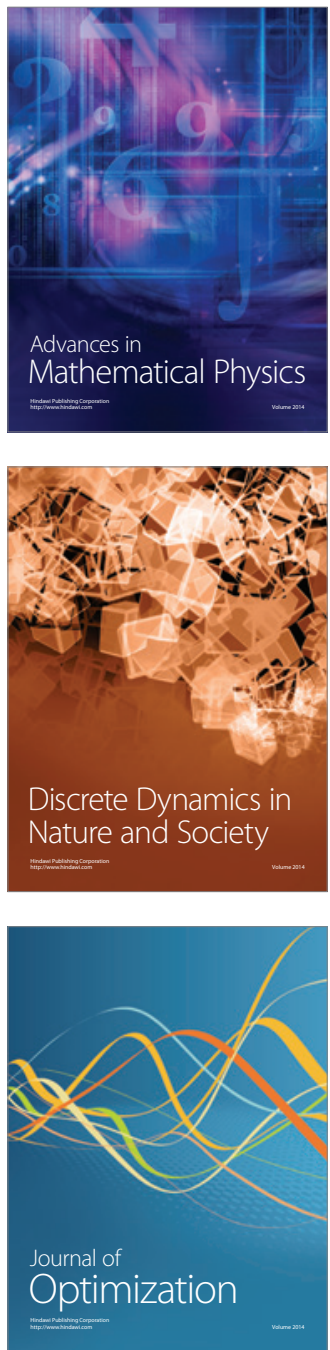